08,10

\title{
Влияние термообработки на формирование активных центров меди, полученных путем взаимодействия хлорида меди с Н-морденитом
}

\author{
() В.В. Срабионян, Г.Б. Сухарина, С.Ю. Каптелинин, В.А. Дурыманов, А.М. Ермакова, \\ Т.И. Курзина, Л.А. Авакян, Л.А. Бугаев
}

Южный федеральный университет,

Ростов-на-Дону, Россия

E-mail: vvsrab@sfedu.ru

Поступила в Редакцию 3 февраля 2020 г.

В окончательной редакции 3 февраля 2020 г.

Принята к публикации 11 февраля 2020 г.

\begin{abstract}
Влияние температурных условий на формирование активных центров меди, полученных путем твердофазного ионного обмена между хлоридом меди и цеолитом Н-морденит изучено с помощью спектроскопии рентгеновского поглощения в околопороговой (XANES) и в протяженной (EXAFS) областях спектров за $K$-краем меди, снятых в in-situ условиях реакции, при температурах, изменяющихся от комнатной до $400^{\circ} \mathrm{C}$. Анализ спектров, выполненный с использованием прямых расчетов CuK-XANES и учета ангармонических поправок для фитинга $\mathrm{Cu} K$-EXAFS, позволил установить, что вплоть до $200^{\circ} \mathrm{C}$ ионный обмен не происходит и медь остается в состоянии $\mathrm{CuCl}$. Повышение температуры более $200^{\circ} \mathrm{C}$ приводит к внедрению атомов меди в каркас цеолита.
\end{abstract}

Ключевые слова: XANES/EXAFS-спектроскопия, цеолиты, Сu-морденит, твердофазный синтез.

DOI: $10.21883 /$ FTT.2020.07.49477.018

\section{1. Введение}

Гетерогенный катализ на основе цеолитов, содержащих атомы переходных металлов, играет все большую роль в химической промышленности. Цеолиты представляют собой микропористые алюмосиликатные соединения с высокой площадью поверхности. Их высокая каталитическая активность обусловлена наличием активных центров, содержащих атомы переходных металлов, чем и объясняется большое количество исследований, посвященных изучению таких центров, в том числе, исследованиям центров в медьсодержащих цеолитах, благодаря их высокой активности в различных химических реакциях. Среди них особый интерес представляет реакция получения метанола из природного газа метана. Существующий на сегодняшний день коммерческий метод получения метанола из метана состоит из двух последовательных этапов: получение синтетического газа, являющегося смесью $\mathrm{CO}$ и $\mathrm{H}_{2}$, и последующего каталитического процесса. Хотя такой путь является относительно эффективным по отношению к выходу метанола, он является достаточно дорогим в реализации и сопряжен с вредными серными загрязнениями. Метод получения метанола из природного газа на основе прямого окисления является значительно менее ресурсоемким (не требует высоких температур и давлений) по сравнению с развитыми на сегодняшний день промышленными технологиями. Трудность реализации такого процесса состоит, однако, в том, что более высокая реактивность получаемого метанола по сравнению с метаном способствует полному его окислению до $\mathrm{CO}_{2}$ и $\mathrm{H}_{2} \mathrm{O}$.
В то же время, существуют работы, в которых показано, что цеолиты ZSM-5 и Мордениты (MOR) стабилизируют мостиковые центры $\mathrm{M}-\mathrm{O}-\mathrm{M}(\mathrm{M}=\mathrm{Fe}, \mathrm{Cu}, \mathrm{Co})$, которые, в частности, способны производить метанол из метана в цеолитах $\mathrm{Cu}-\mathrm{ZSM}-5$ [1,2] и $\mathrm{Cu}$-мордените (Cu-MOR) [3-6] путем активации связей C-H в метане и стабилизации образующихся на начальной стадии интермедиатов. В работах $[3,6]$ показано, что в механизме эффективного превращения метана в метанол важную роль играют ионы $\mathrm{Cu}^{+}$, которые являются промежуточным продуктом в реакции с метаном и способны к расщеплению молекулярного кислорода в процессе формирования активных медных прекурсоров. Метод синтеза таких материалов, используемый при ионном обмене, влияет как на положение ионообменных катионов, так и на их количество. Традиционно реакция ионного обмена проводится суспензированием в водных растворах солей металлов. В процедуре жидкофазного ионного обмена, используемой для приготовления катализатора $\mathrm{Cu}-\mathrm{MOR}$ в работе [7] в реакциях превращения метана в метанол, использовался ацетат меди или нитрат меди в качестве прекурсора для обмена ионов натрия с ионами $\mathrm{Cu}^{2+}$. Было показано, что восстановление ионов $\mathrm{Cu}^{2+}$, происходящее в процессе жидкофазного ионного обмена, не приводит к равномерному распределению ионов $\mathrm{Cu}^{+}$. С другой стороны, использование в качестве прекурсора хлорида меди в реакции жидкофазного ионного обмена в Na-ZSM-5 приводит к очень низким выходам метанола по сравнению с использованием ацетата меди или нитрата меди [1]. Для гомогенного распределения ионов $\mathrm{Cu}^{+}$ в цеолите $\mathrm{Cu}-\mathrm{MOR}$ многообещающим представляется 
метод твердофазного ионного обмена $[8,9]$. В настоящей работе такой обмен осуществляется путем механического смешивания порошков $\mathrm{H}-\mathrm{MOR}$ и соли $\mathrm{CuCl}$ с последующей термической обработкой в потоке газа или в вакууме с целью вызвать газофазную реакцию $\mathrm{Cu}^{+}$с кислотными центрами Бренстеда, содержащими Н [10]. При этом смесь $\mathrm{H}-\mathrm{MOR}$ и $\mathrm{CuCl}$ нагревается до высоких температур с целью получения $\mathrm{Cu}^{+}-\mathrm{MOR}$ и $\mathrm{HCl}$. Последний продукт выделяется и удаляется с газовым потоком.

Целью настоящей работы является изучение влияния температурных условий на формирование активных центров меди и определение локальной атомной структуры таких центров в образцах $\mathrm{Cu}-\mathrm{MOR}$, синтезированных методом твердофазного ионного обмена $\mathrm{CuCl} \leftrightarrow \mathrm{H}-\mathrm{MOR}$ (раздел 2). В разделе 3 представлены результаты определения структуры ближнего окружения атомов меди в образцах $\mathrm{Cu}-\mathrm{MOR}$, полученных при температурах от комнатной (room temperature RT) до $200^{\circ} \mathrm{C}$. Структурный анализ выполнен методами спектроскопии рентгеновского поглощения в околопороговой (XANES) и протяженной (EXAFS) областях спектров [11].

\section{2. Экспериментальные и теоретические методы}

\section{1. Синтез образцов Си-Морденита}

Исследуемые образцы были получены в работе [12]. Для приготовления H-MOR использовалась суспензия Na-MOR ( $\mathrm{Si} / \mathrm{Al}=8.5)\left(10\right.$ g.) в $1 \mathrm{~mol}$ раствора $\mathrm{NH}_{4} \mathrm{NO}_{3}$ в деионизированной воде $(300 \mathrm{ml})$ для обмена $\mathrm{NH}_{4}^{+}$. Рacтвор размешивался и нагревался до $60^{\circ} \mathrm{C}$ в течении 2-х $\mathrm{h}$. После охлаждения суспензию фильтровали и осадок промывали деионизированной водой, затем высушивали при $120^{\circ} \mathrm{C}$ в течении $12 \mathrm{~h}$. Высушенный цеолит был измельчен в очень тонкий порошок, и процедура водного ионного обмена, фильтрации, сушки и дробления была повторена. Затем цеолит был отожжен в потоке $\mathrm{N}_{2}$ при $500^{\circ} \mathrm{C}$ в течении $3 \mathrm{~h}$, после чего получается H-MOR. Для того, чтобы синтезировать $\mathrm{Cu}-\mathrm{MOR}$ методом твердофазного ионного обмена, сначала смешивались порошки $\mathrm{CuCl}(42 \mathrm{mg})$ и $\mathrm{H}-\mathrm{MOR}(0.7 \mathrm{~g})$ и нагревались от комнатной температуры до $120^{\circ} \mathrm{C}$ со скоростью $1^{\circ} \mathrm{C} / \min$ в потоке гелия. Затем, чтобы удалить воду из цеолита, давление понижалось до $2 \mathrm{mbar}$ в течении $1 \mathrm{~h}$. После этого продолжается нагревание в атмосфере гелия до $450^{\circ} \mathrm{C}$ со скоростью $1^{\circ} \mathrm{C} / \mathrm{min}$. Реакция при $450^{\circ} \mathrm{C}$ длилась в течении $40 \mathrm{~h}$.

\subsection{CuK-XAFS измерения и методика обработки спектров}

Для установления влияния термообработки на формирование активных центров меди, полученных путем взаимодействия хлорида меди с цеолитом H-MOR, использовались методы XANES- и EXAFS-спектроскопии.
Спектры рентгеновского поглощения за $K$ краем меди (CuK-XAFS) были измерены на линии SuperXAS Швейцарского источника синхротронного излучения института Пауля Шерера в Виллигене в Швейцарии [13]. Измерения спектров рентгеновского поглощения проводились в in-situ условиях: при комнатной температуре (Cu-MOR_RT), при $120^{\circ} \mathrm{C}(\mathrm{Cu}-\mathrm{MOR} 120)$, при $200^{\circ} \mathrm{C}$ (Cu-MOR_200), при $300^{\circ} \mathrm{C}\left(\mathrm{Cu}-\mathrm{MOR} \_300\right)$ и при $400^{\circ} \mathrm{C}$ (Cu-MOR_400). Измерения приводились в гелиевой атмосфере, кроме измерений при температуре $120^{\circ} \mathrm{C}$, которые проводились при условиях низкого давления. Первичная обработка $\mathrm{Cu} K$-XAFS-спектров, включающая нормировку коэффициента поглощения $\mu(E)$ и последующее выделение из него осциллирующей части $\chi(k)$ в протяженной энергетической области спектра (EXAFS), выполнена с помощью широко распространенного программного пакета Ifeffit $[14,15]$. Анализ фурьеобразов $\mathrm{F}(R)$ экспериментальных $\mathrm{Cu} K$-EXAFS-спектров осуществлялся в шкале межатомных расстояний $(R)$ на основе моделей ближнего окружения атомов меди в исследуемых катализаторах, при рассматриваемых температурах.

\section{3. Результаты и обсуждение}

Сопоставление экспериментальных $\mathrm{Cu} K$-XANES, измеренных в атмосфере гелия при температурах от комнатной (RT) до $400^{\circ} \mathrm{C}$, а также модули Фурье-образов $|\mathrm{F}(R)| \chi(k)$-функций, соответствующих $\mathrm{Cu} K$-EXAFS, в рассматриваемом температурном диапазоне представлены на рис. $1, a-c$ и рис. $1, d-f$, соответственно. Как видно из рис. $1, a, d$ в температурном диапазоне от RT до $200^{\circ} \mathrm{C}$ в локальном атомном окружении меди изменений не происходит, все основные спектральные особенности $A, B, C$ и $D$ в CuK-XANES, и особенности $\mathrm{F}(R)$ в $\mathrm{Cu} K$-EXAFS сохраняются, а наблюдаемое уменьшение величин $\mathrm{F}(R)$ можно объяснить увеличением теплового движения атомов с ростом температуры.

\subsection{Cu-MOR при температурах RT, 120 и $200^{\circ} \mathrm{C}$}

Для определения локальной структуры атомов $\mathrm{Cu}$ в процессе ионного обмена $\mathrm{CuCl} \leftrightarrow \mathrm{H}-\mathrm{MOR}$ при температурах от RT до $200^{\circ} \mathrm{C}$ были выполнены расчеты XANES-спектров за $K$-краем $\mathrm{Cu}$ для наиболее вероятной модели ближнего окружения поглощающего атома $\mathrm{Cu}$ - структуры $\mathrm{CuCl}$. Было выдвинуто предположение, что структура $\mathrm{CuCl}$ должна сохраняться, поскольку температуры в рассматриваемом диапазоне являются достаточно низкими. На рис. 2 представлено сопоставление экспериментального XANES за $K$-краем $\mathrm{Cu}$ в $\mathrm{Cu}-\mathrm{MOR}$ при комнатной температуре (RT) с теоретическим спектром, рассчитанным для $K$-края $\mathrm{Cu}$ в $\mathrm{CuCl}$. Рассчитанный, с использованием программного комплекса FDMNES [16], $\mathrm{Cu} K$-XANES-спектр в $\mathrm{CuCl}$ полностью описывает, представленный в работе [17], 
Основные параметры, характеризующие локальную структуру Сu в образцах MOR_RT, Cu-MOR_120 and Cu-MOR_200

\begin{tabular}{c|c|c|c|c|c|c|c|c}
\hline \multirow{2}{*}{ Образцы } & \multicolumn{2}{|c|}{ Параметры парного потенциала $V(r)$} & \multicolumn{5}{c}{ Параметры связей Сu-Cl } \\
\cline { 2 - 9 } & $\begin{array}{c}a, \\
\mathrm{eV} \cdot \AA^{-2}\end{array}$ & $\begin{array}{c}b, \\
\mathrm{eV} \cdot \AA^{-3}\end{array}$ & $\begin{array}{c}c, \\
\mathrm{eV} \cdot \AA^{-4}\end{array}$ & $S_{0}^{2}$ & $\begin{array}{c}R, \\
\AA\end{array}$ & $\begin{array}{c}\sigma^{2}, \\
\mathrm{~A}^{2}\end{array}$ & $\begin{array}{c}C_{3}, \\
\AA^{3} \cdot 10^{-4}\end{array}$ & $\begin{array}{c}C_{4}, \\
A^{4} \cdot 10^{-5}\end{array}$ \\
\hline Cu-MOR_RT & & & & 0.65 & 2.316 & 0.0070 & 1.33 & -2.7 \\
Cu-MOR_120 & 2.931 & 1.314 & 7.487 & 0.63 & 2.318 & 0.0084 & 4.11 & -6.1 \\
Cu-MOR_200 & & & & 0.61 & 2.321 & 0.0094 & 8.22 & -10.7
\end{tabular}
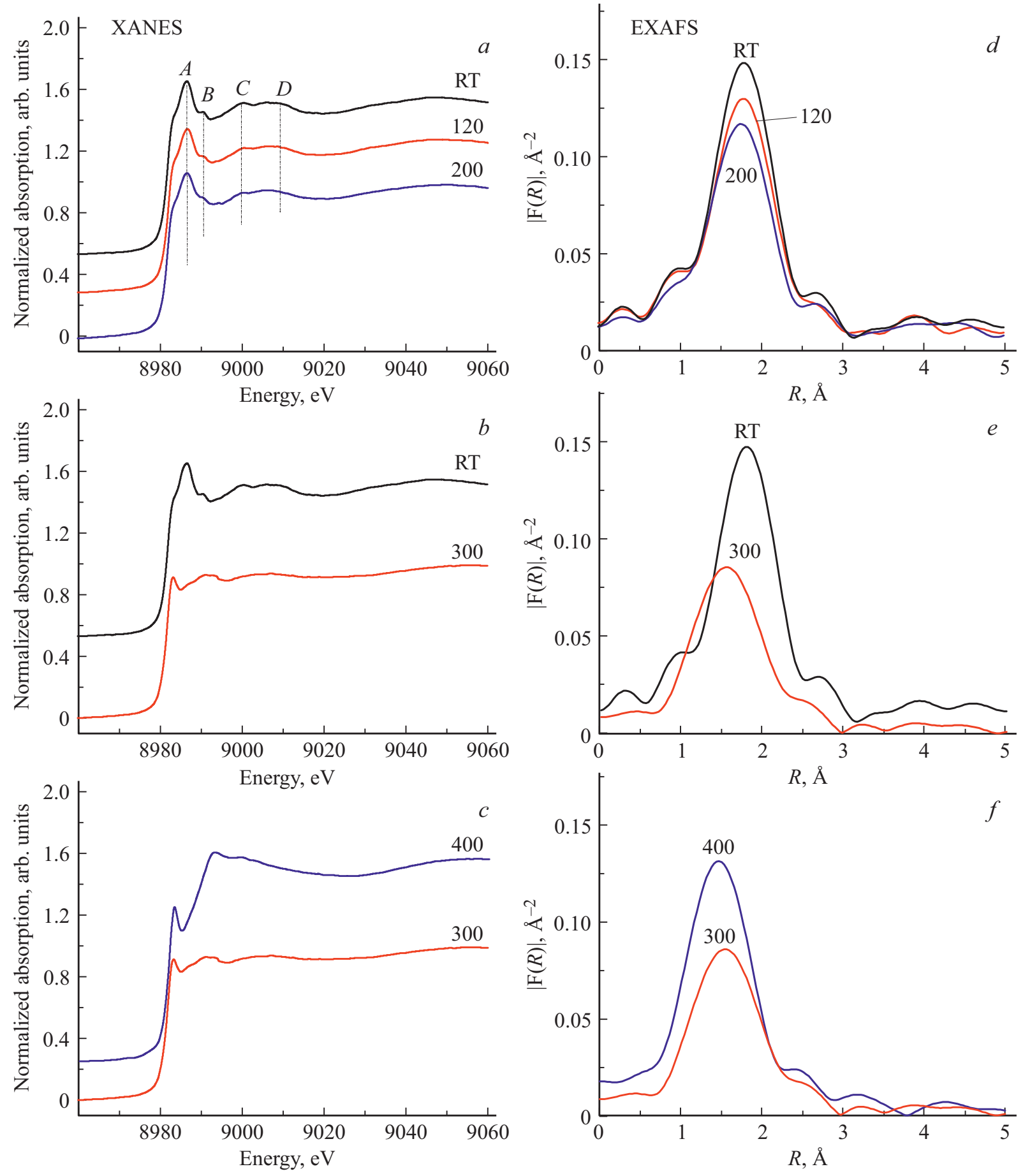

Рис. 1. Сопоставление экспериментальных спектров рентгеновского поглощения XANES/EXAFS за $K$-краем Cu в процессе ионного обмена на разных стадиях синтеза при различных температурах: $R T, 120$ и $200^{\circ} \mathrm{C}(a, d) ; R T$ и $300 \mathrm{C}(b, e) ; 300$ and $400^{\circ} \mathrm{C}(c, f)$. 


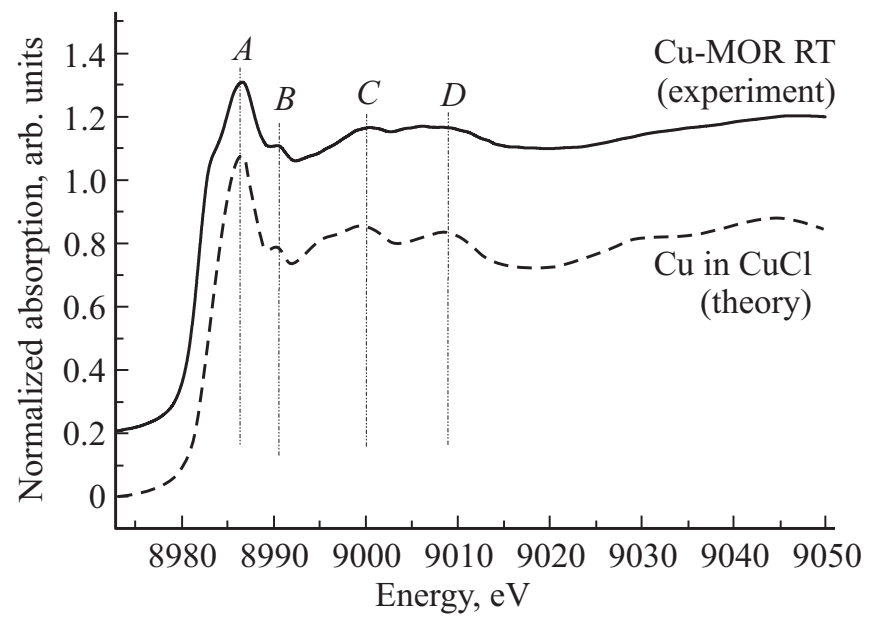

Рис. 2. Сопоставление экспериментального XANES за $K$-краем $\mathrm{Cu}$ в $\mathrm{Cu}$-MOR при комнатной температуре (верхняя сплошная кривая) с теоретическим XANES за $K$-краем Cu в $\mathrm{CuCl}$ (нижняя штриховая кривая)

экспериментальный $\mathrm{CuK}$-XANES-спектр в $\mathrm{CuCl}$. Кроме того, как видно на рис. 2, сопоставление XANESспектров за $K$-краем $\mathrm{Cu}$ позволило сделать вывод, что локальная атомная структура меди в образцах Cu-MOR-RT, Cu-MOR-120 и Cu-MOR-200 идентична локальной структуре меди в $\mathrm{CuCl}$.

На первый взгляд эти вычисления противоречат EXAFS данным рис. 1. В структуре $\mathrm{CuCl}$ вторая сфера поглощающего атома $\mathrm{Cu}$ содержит 12 атомов меди на расстояниях $3.82 \AA[18]$, их вклад должен быть отчетливо виден на $\mathrm{F}(R)$ в EXAFS за $K$-краем меди, однако на рис. 1 мы не наблюдаем ожидаемого результата. Наблюдаемое „противоречие“ объясняется в работе [17], где рассматривается локальное окружение $\mathrm{Cu}$ в $\mathrm{CuCl}$ для температурного диапазона от $-267^{\circ} \mathrm{C}$ до $20^{\circ} \mathrm{C}$. В [17] показано, что вклад второй сферы $\mathrm{Cu}$ в $\mathrm{F}(R)$ отчетливо виден при низких температурах и полностью исчезает при комнатной - RT. Наблюдаемое размазывание вклада от второй сферы при комнатной и более высоких температурах предполагает, что для описания $\mathrm{Cu} K$-EXAFS требуется учет ангармонических эффектов обусловленный тепловым движением атомов, что особенно важно при больших значениях волнового числа фотоэлектрона $(k)$ [19-21].

С этой целью, подгоночная модель локальной структуры $\mathrm{Cu}$ была реализована с учетом ангармонических эффектов, использовался ангармонический эффективный парный потенциал в виде разложения в ряд по значениям расстояний $r=R-R_{0}\left(R\right.$ и $R_{0}-$ мгновенное и равновесное межатомные расстояния соответственно)

$$
U(r)=\frac{a \cdot r^{2}}{2}+b \cdot r^{3}+c \cdot r^{4},
$$

где $a, b$ и $c$ - параметры парного потенциала $V(R)$. В рамках используемого приближения для учета ангармоничности в модельной формуле $\chi(k)$ EXAFS, $a, b$ и $c$ были учтены в виде так называемого кумулянтного разложения с параметрами: $R-$ межатомное расстояние, параметр Дебая-Валлера (DW) $-\sigma_{2}$ и кумулянты $C_{3}, C_{4}[21,22]$. Таким образом, принимая во внимание
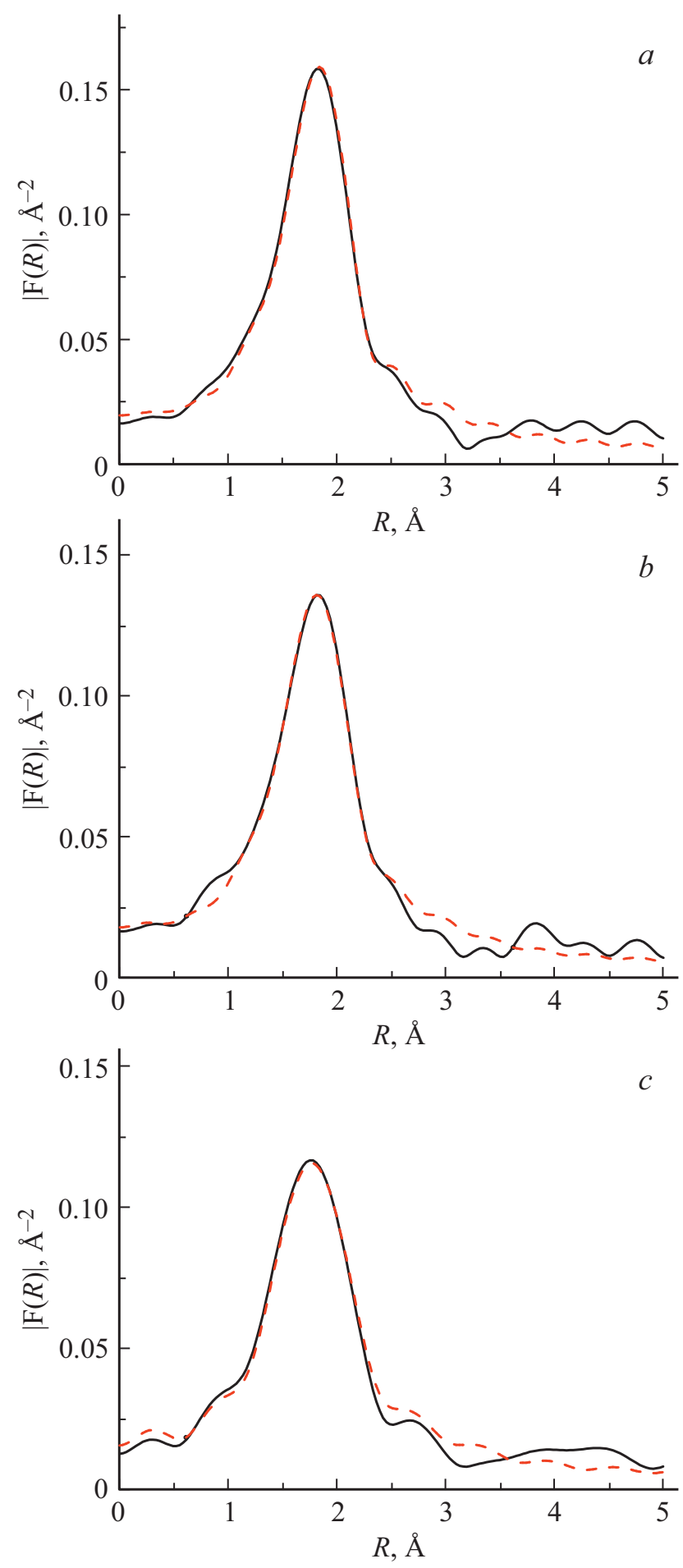

Рис. 3. Сопоставление модулей фурье-образов $|\mathrm{F}(R)|$ $\chi(k)$-функций экспериментального $\mathrm{Cu} K$-EXAFS в цеолите $\mathrm{Cu}-\mathrm{MOR}$ (сплошные черные кривые) с функциями подгонки (штриховые кривые) для образцов: Cu-MOR_RT (a), Cu-MOR_120 (b) и Cu-MOR_200 (c). 
тот факт, что в результате анализа $\mathrm{Cu} K$-края XANESспектров, оказалось, что локальная структура $\mathrm{Cu}$ в $\mathrm{Cu}-\mathrm{MOR}$ при RT, 120 и $200^{\circ} \mathrm{C}$ остается такой же, как в $\mathrm{CuCl}$, подгонка была выполнена одновременно для трех соответствующих спектров $\mathrm{Cu} K$-EXAFS. Такой подход позволил использовать всего восемь варьируемых параметров, в том числе четыре параметра $a, b, c$ и $R_{0}$ при условии их независимости от температуры. Значения параметров, характеризующих локальную структуру $\mathrm{Cu}$ в $\mathrm{Cu}$-цеолите, полученные при этих трех температуpax, представлены в таблице. На рис. 3 показано, что в диапазоне $R \leq 3 \AA$ подгонка, основанная на модели локальной структуры $\mathrm{Cu}$, состоящей из одной оболочки с четырьмя ближайшими атомами $\mathrm{Cl}\left(N_{\mathrm{Cl}}=4\right.$, как в $\mathrm{CuCl})$, позволила воспроизвести все основные особенности „экспериментального“ $\mathrm{F}(R)$. Различия в значениях спектроскопического фактора $S_{0}^{2}$ для трех образцов находятся в пределах погрешности определения точности параметров амплитуды $(\sim 7-10 \%)$ согласно XAFS [23], а сами значения $S_{0}^{2}$ близки по значению к $S_{0}^{2} \sim 0.8$ в $\mathrm{CuCl}$ из [17] и к $S_{0}^{2}=0.78$ в медной фольге, полученному из экспериментальных EXAFS-данных.

\section{4. Заключение}

Проведено рентгеноспектральное исследование влияния температуры на локальную атомную структуру меди в процессе твердофазного ионного обмена между хлоридом меди и цеолитом H-MOR. На основе метода конечных разностей (FDMNES) проведено моделирование околопороговой области спектров рентгеновского поглощения для наиболее вероятной локальной атомной структуры меди, реализуемой в температурном диапазоне от RT до $200^{\circ} \mathrm{C}$. Верификация модели была выполнена на основе EXAFS-анализа с учетом ангармонических эффектов теплового колебания атомов. Полученные результаты позволили сделать вывод, что в трех образцах, соответствующих комнатной температуре, 120 и $200^{\circ} \mathrm{C}$, более 90\% атомов $\mathrm{Cu}$ находятся в фазе $\mathrm{CuCl}$. При повышении температуры до 300 и $400^{\circ} \mathrm{C}$, как XANES, так и EXAFS экспериментальные спектры меди претерпевают кардинальные изменения, свидетельствующие о том, что при изменении температуры от 200 до $300^{\circ} \mathrm{C}$ медь попадает в каркас цеолита, а при температуре от 300 до $400^{\circ} \mathrm{C}$ локальное окружение меди в цеолите претерпевает существенные изменения. Для определения таких структур необходимо использовать подход, основанный на получении наиболее вероятных моделей активного медьсодержащего центра методом теории функционала плотности, с последующим выбором той из моделей, которая обеспечит получение теоретических XANES-спектров меди и фурьеобразов EXAFS в протяженной области межатомных расстояний в согласии с экспериментальными спектрами. Эти исследования будут выполнены в ближайшее время.

\section{Благодарности}

Авторы выражают благодарность профессору Е.А. Бокховену (Швейцарский федеральный институт технологии и биоинженерии, ETH, Zurich) за участие и помощь в работе.

\section{Финансирование работы}

Работа выполнена при поддержке гранта Российского научного фонда (РНФ) по проекту № 18-72-00096 „Изменения атомной и электронной структуры активных

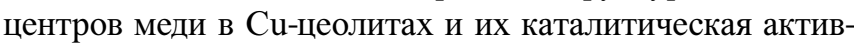
ность в ходе реакций окисления метана до метанола“. Часть авторов: Л.А. Бугаев, Л.А. Авакян, Г.Б. Сухарина, Т.И. Курзина - благодарны Южному фередальному университету за финансовую поддержку.

\section{Конфликт интересов}

Авторы заявляют, что у них нет конфликта интересов.

\section{Список литературы}

[1] N.V. Beznis, B.M. Weckhuysen, J.H. Bitter. Catal. Lett. 138, 14 (2010).

[2] T. Sheppard, C.D. Hamill, A. Goguet, D.W. Rooney, J.M. Thompson. Chem. Commun. (Camb). 50, 11053 (2014).

[3] E.M. Alayon, M. Nachtegaal, M. Ranocchiari, J.A. van Bokhoven. Chem. Commun. 48, 404 (2012).

[4] S. Grundner, M.A.C. Markovits, G. Li, M. Tromp, E.A. Pidko, E.J.M. Hensen, A. Jentys, M. Sanchez-Sanchez, J.A. Lercher. Nature Commun. 6, 7546 (2015).

[5] M.H. Mahyuddin, A. Staykov, Y. Shiota, K. Yoshizawa. ACS Catal. 6, 8321 (2016).

[6] S.E. Bozbag, E.M.C. Alayon, J. Pecháček, M. Nachtegaal, M. Ranocchiari, J. van Bokhoven. Catal. Sci. Technol. 6, 5011 (2016).

[7] E.M.C. Alayon, M. Nachtegaal, M. Ranocchiari, J.A. van Bokhoven. Chim. Int. J. Chem. 66, 668 (2012).

[8] C. Lamberti, S. Bordiga, M. Salvalaggio, G. Spoto, A. Zecchina, F. Geobaldo, G. Vlaic, M. Bellatreccia. J. Phys. Chem. B 101, 344 (1997).

[9] C. Lamberti, S. Bordiga, A. Zecchina, M. Salvalaggio, F. Geobaldo, C. Otero Areán. J. Chem. Soc. Faraday Trans. 94, 1519 (1998).

[10] G. Spoto, A. Zecchina, S. Bordiga, G. Ricchiardi, G. Martra, G. Leofanti, G. Petrini. Appl. Catal. B 3, 151 (1994).

[11] J.A. van Bokhoven, C. Lamberti. X-Ray Absorption and X-Ray Emission Spectroscopy: Theory and Applications (2015).

[12] S.E. Bozbag, E.M.C. Alayon, J. Pecháček, M. Nachtegaal, M. Ranocchiari, J.A. van Bokhoven. Catal. Sci. Technol. 6, 5011 (2016).

[13] P.M. Abdala, O.V. Safonova, G. Wiker, W. van Beek, H. Emerich, J.A. van Bokhoven, J. Sá, J. Szlachetko, M. Nachtegaal. Chim. Int. J. Chem. 66, 699 (2012).

[14] M. Newville. J. Synchrotron Rad. 8, 322 (2001). 
[15] B. Ravel, M. Newville. J. Synchrotron Rad. 12, 537 (2005).

[16] Y. Joly. Phys. Rev. B 63, 125120 (2001).

[17] M. Vaccari, R. Grisenti, P. Fornasini, F. Rocca, A. Sanson. Phys. Rev. B 75, 184307 (2007).

[18] R.W.G. Wyckoff. Crystal Structures. John Wiley, N.Y. (1963).

[19] D.S. Yang, S.K. Joo. Solid State Commun. 105, 595 (1998).

[20] N. Van Hung, N. Ba Duc, R.R. Frahm, J. Phys. Soc. Jpn 72 , 1254 (2003).

[21] J.M. Tranquada, R. Ingalls. Phys. Rev. B 28, 3520 (1983).

[22] P. Fornasini, F. Monti, A. Sanson, J. Synchrotron Rad. 8, 1214 (2001).

[23] L.A. Bugaev, P. Ildefonse, A.M. Flank, A.P. Sokolenko, H.V. Dmitrienko. J. Phys. Condens. Matter 12, 1119 (2000).

Редактор Д.В. Жуманов 